\title{
Discussion on the Characteristics and Symptom Classification of COVID-19 in Chinese Medicine
}

\author{
Na Yang1*, Yun Li', Wangbo Cao², Danan Yang1 \\ ${ }^{1}$ Dezhou People's Hospital, Dezhou 253000, Shandong Province, China \\ ${ }^{2}$ Dezhou Hospital of Traditional Chinese Medicine, Dezhou 253000, Shandong Province, China \\ *Corresponding author: Na Yang, cao800503@163.com
}

\begin{abstract}
Covid-19 (Novel coronavirus pneumonia, NCP) was characterized by fever, dry cough, fatigue, greasy tongue coating and pulsating pulse. According to the literature, with those four symptoms, examination and identification, it is believed that NCP belongs to the category of "pandemic" in Chinese medicine. The main infection is dampness, infection location in triple energizer, the basic pathogenesis is characterized by "dampness, phlegm, blood stasis heat, toxin and deficiency." According to the law of blood transfer of symptom differentiation of triple energizer and weiqi-yingxue the treatment is divided into stages, such as early stage, middle stage, severe stage and recovery stage. Most of the patients were early stage and middle stage, which were the direct transmission of the disease. A small number of patients had the reverse transmission of pericardium and the internal and external detachment. The main method of treatment is to remove infection, remove dampness and detoxify. In addition, to grasp the treatment of early stage and middle stage patients, helps to stop the disease from developing, and it is the key to reduce the criticality of the disease, as well as reduce the mortality.
\end{abstract}

Keywords: COVID-19; Damp virus pandemic; Triple energizer; Symptom classification and treatment; Dezhou

Publication date: July 2021; Online publication: July 31, 2021

\section{Introduction}

New Coronavirus pneumonia, or novel coronavirus pneumonia (NCP), has spread throughout the country since its onset and has begun to spread worldwide. At the beginning of the disease, traditional Chinese medicine participated in the treatment of the pandemic. The treatment rate of traditional Chinese medicine (TCM) in all provinces and cities of China reached more than $90 \%$, and has achieved good treatment effect, as well as accumulated a lot of experiences. NCP is a common disease in Dezhou. For the NCP disease, the author has observed its' pathogenesis, location, treatment and medication selection on 27 cases in the area of Beijing, and has made a preliminary understanding of the characteristics of TCM and the treatment of symptom classification in the treatment of the NCP.

\section{Etiology and pathogenesis}

\subsection{Etiology}

According to Wu Jutong's classification of pandemic-spread diseases, "The pandemic respiratory disease pandemic is raging, and the majority of the cases are filthy. If service is the cause, this is the case in every family." According to Wu Youke's article on pandemic respiratory diseases, "Heat is both the cause and the effect of warmth. Warmth and heat are like the beginning and the finish of a sentence. As a result, heat disease is also known as warm disease. The name pandemic comes from the fact that it passes from family to family like a corvee, as was needed by the government at the time." "A pandemic is when a sickness 
caused by the entry of a form of qi that emerges at a period of time and effects multiple nations, rather than natural phenomena caused by wind, coldness, hotness, and wetness." Combined with the specific discussion in the above literature, the NCP infection is mainly caused by respiratory droplets and close contacts of infected patients. It is highly infectious, with similar symptoms and widespread susceptibility. Therefore, the diagnosis and treatment plan for NCP (trial version sixth) points out that the disease belongs to the category of "pandemic disease" of TCM, and it is one of the plagues of $2019-\mathrm{nCoV}^{[1]}$.

From November 2019 to December 2019, the temperature in Wuhan was higher than that in the same period of the year. After December, there was a continuous overcast and rainy weather, and there was a long term of cooling weather, which led to the appearance of cold and humid climate. The NCP is characterized by fever, dry cough, fatigue, plump, pale or light red tongue with whitish or yellow greasy coating, and slippery or soft pulse. It shows that the nature of pandemic disease is dampness, and the basis of pathological changes is damp pathogen ${ }^{[2]}$.

Damp pathogen causes a wide range of diseases, ranging from skin and muscle surface to internal organs, muscles and bones. According to Wu Jutong's study on respiratory diseases, it is stated that "heat related disease starts from top hand-taiyin to down the lung which is connected to the nose. Therefore, the NCP patients have cough and symptoms of damp pathogen which can cause lung damage. If the damp pathogen is heavy and stays in the central area, the body is prone to fatigue and other symptoms. Sticky and stagnant, damp pathogen, Liu Baoyi's "Warm on the Source" says, "I don't know that the pathogen of pandemic heat disease is absorbed from the mouth and nose, and the pathogen of dampness and filth of the recipient is hidden in the source of the infection, then develops into symptoms of fever, chest distress and nausea." The infection causes fever, nausea, anorexia, diarrhea and other gastrointestinal symptoms. It can be seen that damp pathogens runs through the whole course of the disease. As for the attribute of cold and heat, according to the region, climate, physique and other reasons, there are many concurrent symptoms, such as cold and dampness, damp heat, blood stasis, phlegm, etc.

\subsection{Location and pathogenesis of the disease}

Regarding the location and pathogenesis of NCP, Professor Wang Yuguang and Professor Xiong Jibai, suggests that it is at the lung, Ding, Yuan, Peng Bo and other experts suggests that it is at the spleen; Professor Wang Xinlu suggests that it is at the lung and spleen. The author thinks that the location of the disease is triple energizer. According to Wu Jutong's study on respiratory diseases, "all heat related diseases begin with upper energizer and end in Taiyin." When the lung disease is transmitted inversely, it is diagnosed as a pericardium, where the upper energizer is out of treatment, the middle energizer is transmitted, and finally the lower energizer is transmitted. It is also said that "Taiyin is golden, which warms the qi of fire, and wind is the mother of fire. Thus, the disease begins here, and it must be concluded from Hejian triple energizer." The pandemic gas enters from the mouth and nose and invades the lung health. The spleen is seen as wet soil, in which water and soil absorb mutually. It is straight in the middle energizer, which affects the transportation of water, grain and liquid. The triple energizer is not conducive to gasification, which makes water stagnant. Phlegm and dampness are endogenous. Phlegm and dampness block the operation of qi and blood. Qi stagnation leads to blood stasis and endogenesis. Phlegm, dampness, blood stasis and toxin are combined with each other, which further aggravates the disease. With the progress of the disease, it may appear that it can travel to the liver and kidney "If the middle energizer is not treated, it can travel to the lower energizer, as well as the liver and kidney." Dampness is yin pathogen, which damages the Yang Qi of internal organs and leads to the decline of body functions. Second, the virus was transmitted to the pericardium, invading the blood, causing heart and critical damage. Both of them are 
critical diseases. In addition, it is said in Suwen, Linglan Midianlun "the triple energizer is the officer in charge of the waterway in the body and can guarantee water metabolized normally. Modern scholars believe that NCP is associated with the systemic lymphatic system, and triple energizer is impaired, which is consistent with the decrease of lymphocyte count in patients with NCP ${ }^{[3-6]}$.

It can be seen that this disease is located in triple energizer, which starts from lung wei, then spreads to middle energizer, and finally to lower energizer. The main cause of the disease is dampness, which spreads through the whole process of the disease, and also includes phlegm, blood stasis, heat, toxin and deficiency. The pathogenesis is deficiency of healthy qi, excess of pandemic qi, accumulation in the lung, followed by dampness trapped in the spleen and heat from damp stagnation, disease progression, deficiency and excess.

\section{Treatment and prescription selection}

The principle of treatment based on triple energizer should be "promoting the upper part, clearing the middle part and permeating the lower part" of the lung. According to different regions and different periods of disease, the principle of three factors and symptom classification should be adopted ${ }^{[7]}$.

At the initial stage of the disease, the patients with mild and common type are asymptomatic or have fever and cough, accompanied by white and greasy tongue coating and fluctuating pulse. At this time, the dampness pathogen invades the lung or dampness toxin damages the lung, the lung loses its circulation and function, causing coughing, and the stomach qi is stagnated, which leads to fever. Therefore, the treatment is appropriate to spread the dampness pathogen through the lung and cure the dampness with aromatized medicine. Recommended prescription: Ma-huang, gypsum, almond, notopterygium, Agastache rugosum, pelan, Atractylodes lancea, Poria cocos, Atractylodes macrocephala, Magnolia officinalis, Cao-guo, betel nut, licorice.

With the deepening of pathogenic factors, some common type patients entered the middle stage of the disease, with symptoms of aggravating cough, dry cough or expectoration, fatigue, nausea, diarrhea, anorexia, white greasy or yellow greasy tongue coating, and fluctuating pulse. At this time, it is the same as dampness and toxin attacks the lung, pathogens enter the middle energizer, spleen and stomach doesn't function properly, phlegm is endogenous, phlegm is blocking the water channel, affecting the operation of water, and the worsens the situation, water dampness and phlegm is aggravating. For the patients with cold, it is better to open the membrane and remove dampness and Phlegmpinellia ternata, Magnolia officinalis, Poria cocos, Perilla frutescens, Cortex mori, Cortex rehmanniae, Radix Glycyrrhizae, Fritillaria thunbergii, Agastache rugosa, Areca catechu, and Cao-gou. It is suggested that the following prescriptions should be used for the treatment of respiratory diseases. Ma-huang, almond, gypsum, licorice, Ting $\mathrm{Li} \mathrm{Zi}$, Trichosanthes, earthworm, coix seed, Atractylodes lancea, Cortex mori, Cortex rehmanniae, Rhizoma atractylodis, Poria cocos, Magnolia officinalis. For the patients with severe digestive tract symptoms, diuresis and turbidity are recommended, cardamom, Agastache rugosa, Artemisia capillaris, talc, Tong cao, Acorus tatarinowii, Atractylodes macrocephala, Poria cocos, plantain seed.

A small number of patients cannot cut off the disease, thus it develops severely. At this time, as there is heat in the blood, it makes it easy for pathogens to stick. The treatment should be focused on clearing the pathogen. treatment should be heat detoxification, blood stasis. Recommended prescription, Ma-huang, almond, gypsum, licorice, agastache, Magnolia officinalis, Cao-guo, Ting Li Zi, Pinellia ternata, Poria cocos, Ophiopogon japonicus, Polygonatum odoratum, Trichosanthes, earthworm, Paeonia lactiflora, Cortex moutan.

After treatment, the symptoms of respiratory tract and digestive tract gradually relieved, lymphocytes 
gradually increased, the scope of inflammation of lung CT gradually narrowed, the detection of viral nucleic acid turned negative, and the disease entered the recovery period. But at this time, the patient may still have fatigue, malaise, slight cough and other symptoms, which is "healthy qi damage, remaining pathogens were not cleared," mainly because the pathogenesis is damp pathogens, which also shows the characteristics of damp disease. At this stage, the main treatment should be strengthening the body and tonifying the lung and spleen. Radix pseudostellariae, Rhizoma atractylodis Macrocephalae, Poria cocos, fried licorice, Pinellia ternata, tangerine peel, Agastache rugosa, Gardenia jasminoides, fried chicken gizzard, Ophiopogon japonicus, Cimicifuga, Scutellaria baicalensis. At the same time, it can be combined with Baduanjin and other physiotherapy methods to regulate the qi and blood of triple energizer, which is conducive to the recovery of the body ${ }^{[8]}$.

Some of them are Shandong people who live, work and study in Wuhan, and some of them are diagnosed patients in Wuhan. The common type was the main type, and the severe and critical type were few. Dezhou has a typical northern climate. In winter, the climate is dry and the room is warm. It is easy to turn dampness into heat, dryness and yin. Therefore, in the process of treatment, the drugs of nourishing yin and moistening dryness were added, such as Ophiopogon japonicus, Shashen, etc.

\section{Conclusion}

The outbreak of NCP is a category of "pandemic" in TCM. It is caused by damp pathogen, seasonal, regional and crowd susceptibility. "Damp" pathogens spread through the whole course of the disease, and the disease is located in triple energizer. The pathogenesis is lack of vital qi, pandemic qi is abundant, the lung is obstructed, and then the dampness is trapped in the spleen causing decline in body fuction. Through the clinical observation of 27 cases of NCP in Dezhou City, combined with triple energizer and stomach qi, Ying blood symptom classification, the treatment of this disease is divided into initial stage, middle stage, severe stage and recovery stage, and good therapeutic effect is obtained.

These are the authors experiences in the treatment of patients during the designated hospitals. I would like to make a brief summary of them for your reference so as to make a contribution to the treatment of NCP with TCM at this very time.

\section{Disclosure statement}

The author declares no conflict of interest.

\section{References}

[1] 2020, New Coronavirus Pneumonia Diagnosis and Treatment Plan (Trial Version Sixth). Tianjin Chinese Medicine, (6):1-5.

[2] Cai MY, Wu P, Yang RX, Peng B, 2020, Application of "Lung and Spleen Simultaneous Treatment" in New Coronavirus Pneumonia. Chinese Pharmacology and Clinic, (3):1-9.

[3] Miao Q, Cong XD, Wang B, et al., 2020, Understanding and Thinking of Traditional Chinese Medicine in New Coronavirus Pneumonia. Chinese medicine magazine, 61(4): 286-288.

[4] Xiong JB, Xiong JB, 2020, Talk About the Hunan Traditional Chinese Medicine Diagnosis and Treatment Plan for New Coronavirus Pneumonia. Journal of Hunan University of Chinese Medicine, 40 (2): 123-128. 
[5] Ding RC, Wang F, Lu H, et al., 2020, Discussion on Diagnosis and Treatment of New Coronavirus pneumonia from Spleen and Stomach. Chinese Journal of Traditional Chinese Medicine, (2):1-8.

[6] Jin Y, Wang XL, 2020, TCM Treatment of New Coronavirus Pneumonia in Shandong. Shandong Journal of Traditional Chinese Medicine, (2):1-4.

[7] Feng RQ, Lu T, Li B, 2020, “Three Reasons for Adaptation” Theory to Treat New Coronavirus Pneumonia. Chinese Journal of Traditional Chinese Medicine, (2):1-13.

[8] Zheng WK, Zhang JH, Yang FW, et al., 2020, Treatment of Respiratory Diseases of New Coronavirus Infection from Damp Pandemic Disease. Chinese Medicine Magazine, (6):1-5. 